\title{
Differential diagnosis between newly diagnosed asthma and COPD using exhaled breath condensate metabolomics: a pilot study
}

To the Editor:

Asthma and chronic obstructive pulmonary disease (COPD) are heterogeneous diseases with high pathological burden and healthcare costs [1-3]. In outpatient clinical practice, an accurate differential diagnosis is often very difficult, particularly in adult smokers, requiring specific lung function tests $[4,5]$. Since nuclear magnetic resonance (NMR)-based metabolomics of exhaled breath condensate (EBC) discriminates adults with COPD [6-8] or asthma [9] from healthy subjects, we hypothesised that it is also able to differentiate asthma and COPD patients of different severities.

After approval by the Maugeri ethics committee, we recruited prospectively patients with a new diagnosis of asthma $(n=31)$ and COPD $(n=44)$ according to current Global Initiative for Asthma and Global Initiative for Chronic Obstructive Lung Disease guidelines. Six patients with asthma and nine COPD patients were excluded because of relevant comorbidities that could potentially affect the analysis (nine for the presence of coronary or valvular heart disease, five for the presence of diabetes mellitus, one for hypothyroidism). In addition, the EBC samples obtained from five asthma and three COPD subjects were technically unsuitable for NMR analysis. The final 20 asthma and 32 COPD subjects were used to build the reference statistical model (table 1). Since no a priori analysis was possible, we could only evaluate the adequacy of our sample size a posteriori, estimating a sample size of $17 \pm 3$ asthma and $23 \pm 3$ COPD patients. A second cohort was also enrolled for an external blind validation comprising 13 asthma and 20 COPD patients.

EBC collection was achieved with a TURBO-DECCS condenser (Medivac, Pilastrello, Italy) as reported [10]. NMR spectra were recorded at $27^{\circ} \mathrm{C}$ in a randomised sequential order on a $600-\mathrm{MHz}$ Bruker Avance-III spectrometer (Bruker BioSpin GmbH, Rheinstetten, Germany) equipped with a CryoProbe using standard experiments. Metabolites were identified by resorting to two-dimensional experiments. Within-day, between-day, and technical repeatability, and detection limit were assessed as reported [7, 9].

Proton NMR spectra were automatically data reduced to 390 integral segments ("buckets"), each of $0.02 \mathrm{ppm}$, using the Bruker AMIX 3.6 software package. Unsupervised principal component analysis (PCA) was first applied. However, to better identify clustering, we used orthogonal projections to latent structures discriminant analysis (OPLS-DA), and the obtained model showed improved predictive and interpretive abilities, and in a permutation test $(n=300)$ revealed no overfit. The model quality was evaluated via the goodness-of-fit $\left(\mathrm{R}^{2}\right)$ and the goodness-of-prediction $\left(\mathrm{Q}^{2}\right)$ parameters [11]. Metabolite quantification was obtained using the corresponding normalised buckets. Metabolite statistical significance was determined by parametric ( $\mathrm{t}$-test) or non-parametric (Mann-Whitney U-test) tests according to the results of a normality test performed to evaluate each distribution (Shapiro-Wilk, Kolgomorov-Smirnov test). p-values $<0.05$ were considered as statistically significant.

All EBC classes were homogeneous, as PCA did not detect any subgroup related to the clinical characteristics reported in table 1. OPLS-DA analysis of EBC profiles differentiated asthma and COPD with strong regression (95\%, $\mathrm{p}<0.0013$; figure 1a) and high-quality parameters $\left(\mathrm{R}^{2}=0.86\right.$ and $\mathrm{Q}^{2}=0.86$ ). COPD patients, compared with asthma patients, show an increase in ethanol (mean \pm sD $25.56 \pm 4.57 \mu \mathrm{M}$ versus $12.15 \pm 3.12 \mu \mathrm{M} ; \mathrm{p}=0.0119)$ and methanol $(10.67 \pm 2.99 \mu \mathrm{M}$ versus $5.01 \pm 2.02 \mu \mathrm{M} ; \mathrm{p}=0.049)$, and

@ERSpublications

NMR profiling of EBC discriminates between asthma and COPD and this may help clinicians with differential diagnosis http://ow.ly/cNGI30hWYAE

Cite this article as: Maniscalco M, Paris D, Melck DJ, et al. Differential diagnosis between newly diagnosed asthma and COPD using exhaled breath condensate metabolomics: a pilot study. Eur Respir J 2018; 51: 1701825 [https://doi.org/10.1183/13993003.01825-2017]. 
TABLE 1 Clinical characteristics of the subjects recruited to the study $\#$

\begin{tabular}{|c|c|c|c|c|c|c|}
\hline & Asthma & COPD & p-value & Asthma validation set & COPD validation set & p-value \\
\hline \multicolumn{7}{|l|}{ Anthropometric data } \\
\hline Subjects & 20 & 32 & & 13 & 20 & \\
\hline Sex (females/males) & $11 / 9$ & $10 / 22$ & 0.40 & $7 / 6$ & $4 / 10$ & 0.80 \\
\hline $\mathrm{BMI}^{+} \mathrm{kg} \cdot \mathrm{m}^{-2}$ & $22.7 \pm 2.9$ & $24.4 \pm 6.1$ & 0.25 & $23.3 \pm 3.8$ & $25.1 \pm 5.3$ & 0.30 \\
\hline Current smokers & 5 & 7 & 0.90 & 3 & 8 & 0.70 \\
\hline Time of quitting years & $3.5 \pm 2.2$ & $2.5 \pm 1.2$ & 0.04 & $2.8 \pm 1.2$ & $2.1 \pm 1.1$ & 0.53 \\
\hline Smoking history pack-years & $19.5 \pm 2.1$ & $39.0 \pm 15.1$ & $<0.001$ & $14.1 \pm 1.9$ & $37 \pm 12$ & $<0.001$ \\
\hline \multicolumn{7}{|l|}{ Lung function } \\
\hline Pre-BD FEV $1 \%$ pred & $77.3 \pm 13.8$ & $69.8 \pm 7.0$ & 0.027 & $76.1 \pm 10.1$ & $69.2 \pm 7.3$ & 0.027 \\
\hline Pre-BD FVC \% pred & $91.2 \pm 9.9$ & $85.1 \pm 9.0$ & 0.015 & $90.1 \pm 9.8$ & $88.6 \pm 9.0$ & 0.54 \\
\hline Post-BD FEV $1 \%$ pred & $87.7 \pm 9.8$ & $71.3 \pm 7.7$ & $<0.001$ & $89.1 \pm 8.2$ & $72.2 \pm 7.8$ & $<0.001$ \\
\hline \multicolumn{7}{|l|}{ Atopy } \\
\hline Yes/no & $16 / 4$ & $4 / 28$ & 0.004 & $10 / 3$ & $3 / 21$ & 0.04 \\
\hline \multicolumn{7}{|l|}{ Treatment ongoing } \\
\hline No treatment & 7 & 4 & & 5 & 2 & \\
\hline Short acting $\beta_{2}$-agonist & 11 & 13 & & 5 & 10 & \\
\hline Long-acting $\beta_{2}$-agonists & 2 & 7 & & 3 & 7 & \\
\hline Long-acting antimuscarinic antagonist & 0 & 8 & & 0 & 5 & \\
\hline Antihypertensive drugs & 8 & 21 & & 3 & 17 & \\
\hline Statins & 1 & 5 & & 1 & 8 & \\
\hline
\end{tabular}

Data are expressed as $\mathrm{n}$ or mean $\pm \mathrm{SD}$ after assessing for normality with the D'Agostino-Pearson omnibus normality test, unless otherwise stated. Normally distributed values were compared by using the unpaired t-test. If the normality test failed, the Wilcoxon-Mann-Whitney test was used. Group differences were explored by means of 1-way ANOVA, followed by post hoc multiple comparisons according to the Tukey test. Intraclass correlation analysis was performed for each group to estimate the reliability of single measurements. Chi square was used for comparing proportions. Statistical significance was defined as $\mathrm{p}<0.05$. \#: diagnosis was achieved according to current Global Initiative for Asthma (ginasthma.org) and Global Initiative for Chronic Obstructive Lung Disease (goldcopd.org) guidelines. All chronic obstructive pulmonary disease (COPD) and asthma patients were clinically stable. Former smokers had stopped smoking for at least one year. None of the patients was on regular systemic or inhaled corticosteroid, theophylline, antibiotics, antioxidants or mucolytic treatment. Asthma therapies remained unchanged for 3 months before the study. The study excluded all subjects with a respiratory infection within the previous 3 weeks, chronic heart diseases, diabetes mellitus, hypo/hyperthyroidism, the presence of mental or physical disability precluding informed consent or compliance with the protocol, and pregnancy. The final cohorts included 20 asthmatic and 32 COPD patients, which were used to build the model, and 13 asthmatic and 20 COPD patients for the external blind validation. ": age range: asthma, 35-54 years; COPD, 44-67 years. Validation set: asthma, 34-55 years; COPD, 45-68 years. ${ }^{+}$: body mass index (BMI) range: asthma, $18.2-24.8 \mathrm{~kg} \cdot \mathrm{m}^{-2} ; \mathrm{COPD}, 20.3-26.4 \mathrm{~kg} \cdot \mathrm{m}^{-2}$. Validation set: asthma, $17.0-25.2 \mathrm{~kg} \cdot \mathrm{m}^{-2}$; COPD, $19.3-26.1 \mathrm{~kg} \cdot \mathrm{m}^{-2}$. BD: bronchodilator; FEV1: forced expiratory volume in $1 \mathrm{~s}$; FVC: forced vital capacity.

significantly lower levels of formate $(2.63 \pm 0.97 \mu \mathrm{M}$ versus $6.97 \pm 1.12 \mu \mathrm{M} ; \mathrm{p}=0.009)$ and acetone/acetoin $(5.84 \pm 1.49 \mu \mathrm{M}$ versus $12.53 \pm 3.01 \mu \mathrm{M} ; \mathrm{p}=0.0004)$. The area under the curve (AUC) of the receiver operating characteristic (ROC) curve yielded a value of 0.99 for the model of figure 1a.

The relevance of the model was evaluated using a separate cohort (13 asthma and 20 COPD patients, table 1), which was tested blindly. Its projection on the above statistical model generated the scores of figure $1 \mathrm{~b}$. The OPLS-DA classifications shows a strong regression $(95 \%, \mathrm{p}<0.0011)$ and high-quality parameters $\left(\mathrm{R}^{2}=0.89\right.$ and $\left.\mathrm{Q}^{2}=0.87\right)$, therefore confirming that the model obtained from the training set is actually valid. It correctly identified 12 of 13 asthma patients (92.3\% accuracy) and 19 of 20 COPD subjects $(95.0 \%$ accuracy, $5.0 \%$ false-positive results), showing a sensitivity (true positive rate) of $92.3 \%$, and a specificity (true negative rate) of $95.0 \%$. The positive predictive value (probability that the disease is present when the test is positive) is $92.3 \%$, and the negative predictive value (probability that the disease is not present when the test is negative) is $95.0 \%$. The discriminating metabolites were confirmed to be in COPD increased ethanol $(26.31 \pm 5.01 \mu \mathrm{M}$ versus $11.38 \pm 3.98 \mu \mathrm{M} ; \mathrm{p}=0.0101)$ and methanol $(11.43 \pm 3.34 \mu \mathrm{M}$ versus $4.82 \pm 1.79 \mu \mathrm{M} ; \mathrm{p}=0.039)$, and decreased formate $(2.97 \pm 0.69 \mathrm{M}$ versus $7.45 \pm 1.99 \mu \mathrm{M} ; \mathrm{p}=0.003)$ and acetone/ acetoin $(6.04 \pm 1.89 \mu \mathrm{M}$ versus $14.11 \pm 3.87 \mu \mathrm{M} ; \mathrm{p}=0.0009)$. The AUC of the ROC for the total model of figure $1 \mathrm{~b}$ was 0.96 . 

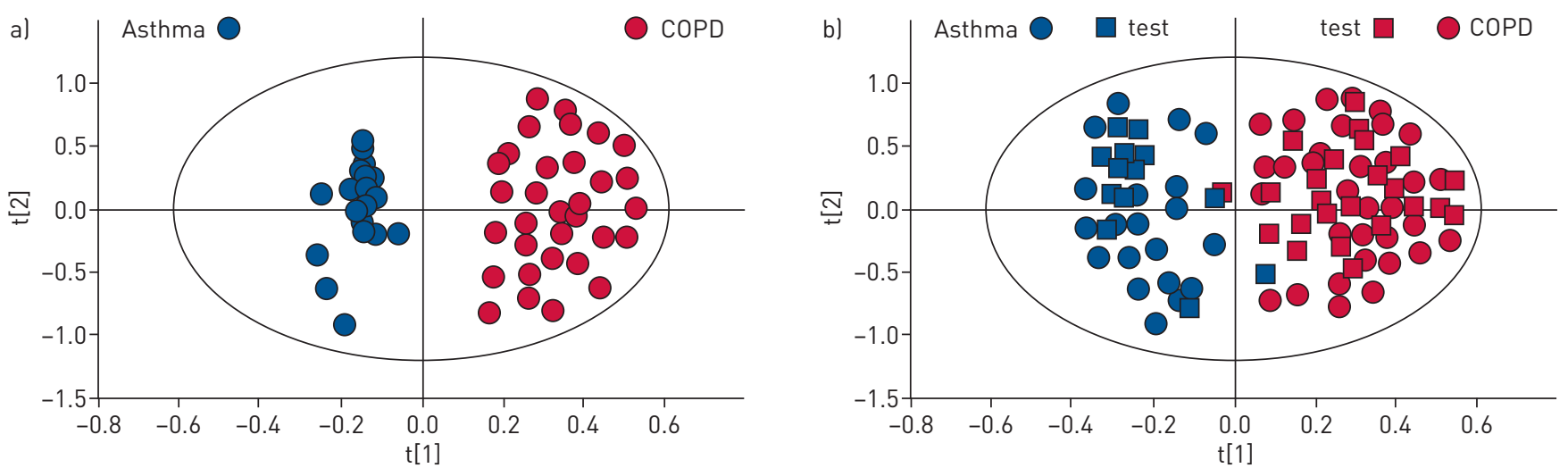

FIGURE 1 Orthogonal projections to latent structures discriminant analysis (OPLS-DA) of exhaled breath condensate (EBC) samples. a) Score plot showing the degree of separation of the model between asthmatic (blue circles) and chronic obstructive pulmonary disease (COPD) (red circles) patients (the training set). The model presents strong regression $(95 \%, p<0.0013)$ and high-quality parameters $\left(R^{2}=0.86\right.$ and $\left.\left.Q^{2}=0.86\right) . b\right) P r e d i c t e d$ scores plot representing classification of the validation cohort obtained by projecting validation samples onto the model assessed by the training set. Circles represent the training set samples (asthma, blue circles; COPD, red circles), while squares refer to the validation set samples. The OPLS-DA classifications of both sets presents a strong regression $(95 \%, p<0.0011)$, with high-quality parameters $\left(R^{2}=0.89\right.$ and $\left.Q^{2}=0.87\right)$. In both plots, the labels $\mathrm{t}[1]$ and $\mathrm{t}[2]$ along the axes represent the scores (the first two partial least-squares components) of the model, which are sufficient to build a satisfactory classification. The models were obtained by applying statistical analysis to nuclear magnetic resonance (NMR) spectra of EBC. Proton spectra were automatically data reduced to integrated regions ("buckets") of 0.02-ppm width (the ppm identifies the position of the NMR line in a spectrum with respect to a reference) using AMIX 3.6 software package. The bucketing data were imported into SIMCA-P+14 package, and principal components analysis and OPLS-DA were performed.

Methanol is metabolised to formaldehyde, which shows a pro-inflammatory action in cells and animal models. Interestingly, methanol concentration increases in lung cancer patients, and notably COPD is characterised by an increased risk of lung carcinoma [12]. Asthma, instead, presents a weaker risk of lung cancer in nonsmokers, and shows high levels of formate. This may be related to the in vitro antiproliferative effect on lung cancer cell lines of formate [13], which, on the contrary, decreases in the sputum of lung cancer patients [14].

Low acetone was observed in the EBC of stable COPD patients compared to healthy nonsmoking and former-smoker subjects with normal lung function [7]; on the contrary, increased level was reported in the exhaled breath of stable COPD patients compared to nonsmoking and smoking subjects with normal lung function [15]. These contrasting results may simply reflect the different sampling techniques. Ethanol and its metabolites are involved in the pathogenesis and progression of COPD [16]. In addition, the main metabolite of ethanol, acetaldehyde, may contribute to the increased risk of lung cancer in COPD [12].

The source of acetoin in our asthma or COPD patients is unknown. It may be the product of the detoxication process of acetaldehyde [17], but may also be a bacterial product produced by both pathogenic and non-pathogenic bacteria [18].

This is the first report demonstrating that the NMR metabolic profiling of EBC can be used to discriminate asthma patients from COPD patients, even in smoking adults, in excellent agreement with the use of an electronic nose [19] or urine metabolomics [20]. Furthermore, the EBC metabolic phenotype ("metabotype") differentiates asthma and COPD better than, for example, a panel of sputum cytokines [21]. The fact that, in our validation cohort, the model correctly attributed both smoking and nonsmoking asthma patients to the "asthma area" (figure 1b), implies that smoking is not the major factor in the metabolomics differentiation of asthma and COPD. In addition, as clinically expected, significantly more atopic subjects are present in the asthma group. However, the possibility that changes in the NMR profiling of asthma EBC may simply be a fingerprinting of atopy was excluded because of the absence of a related subgroup in the found models.

A potential strength of our study is the use of a validation cohort, since the external validation is the only discriminatory evidence that a calculated model can be clinically valuable, regardless of the reported predictive indices. Additionally, several measures were taken for quality control of the data. For EBC collection we minimised the external influence and contamination, and good within-day, between-day, and technical repeatability were observed. Moreover, no influence of demographic parameters was observed. All patients were well characterised according to current international guidelines, and the differences between the groups with respect to age and lung function parameters reflect those expected from the clinical characteristics of asthma and COPD. 
Our study, however, also presents some limitations. First, although the number of samples used is larger than that suggested by backward analysis, the sample size was relatively small. Nevertheless, we were able to distinguish COPD and asthma, identifying subtle differences in the metabolomic pattern.

Second, our cohort is not representative of the whole spectrum of asthma and COPD patients observed in clinical practice. We need to investigate older patients, those with more history of smoking and those with more severe asthma. Likewise, the potential influences of specific drugs (glucocorticoids, theophylline, antileukotrienes, oxygen, antioxidants and antibiotics) on the discriminating power of this approach should also be evaluated. The metabotype potential changes during uncontrolled asthma and symptomatic COPD, and during asthma and COPD exacerbations of different severities should similarly be considered.

Third, future studies should include other control groups such as smokers with normal lung function and patients with bronchiectasis of different aetiologies. We also recognise that a single biological fluid, as the $\mathrm{EBC}$, may not represent the complexity of the metabolic pathways involved in the pathogenesis of these diseases [22].

For all these reasons, we are currently undertaking a comparative study on the metabolic profile of multiple biological fluids (serum, urine, EBC and saliva) obtained from a larger population of COPD and asthma patients, and incorporating the above-mentioned control groups. Notwithstanding the described limitations, we have shown that NMR profiling of EBC discriminates asthma and COPD patients with high sensitivity and specificity, and this may help the clinicians to decrease the number of incorrect diagnosis.

Mauro Maniscalco ${ }^{1,6}$, Debora Paris ${ }^{2,6}$, Dominique J. Melck ${ }^{2,6}$, Antonio Molino ${ }^{3}$, Mauro Carone ${ }^{4}$, Paolo Ruggeri ${ }^{5}$, Gaetano Caramori $\odot^{5}$ and Andrea Motta ${ }^{2}$

${ }^{1}$ Pulmonary Rehabilitation Unit, ICS Maugeri SpA, IRCCS, Telese Terme, Italy. ${ }^{2}$ Institute of Biomolecular Chemistry, National Research Council, Pozzuoli, Italy. ${ }^{3}$ Dept of Respiratory Medicine, Monaldi Hospital (Naples), Naples, Italy. ${ }^{4}$ Pulmonary Rehabilitation Unit, ICS Maugeri SpA, IRCCS, Cassano delle Murge, Italy. ${ }^{5}$ Unità Operativa Complessa di Pneumologia, Dipartimento di Scienze Biomediche, Odontoiatriche e delle Immagini Morfologiche e Funzionali (BIOMORF), Università di Messina, Messina, Italy. ${ }^{6} \mathrm{M}$. Maniscalco, D. Paris and D.J. Melck contributed equally to this manuscript.

Correspondence: Andrea Motta, Institute of Biomolecular Chemistry - CNR, Comprensorio Olivetti, Edificio A, Via Campi Flegrei 34, 80078 Pozzuoli (NA), Italy. E-mail: andrea.motta@icb.cnr.it

Received: July 292017 | Accepted after revision: Dec 282017

Conflict of interest: None declared.

\section{References}

1 Hizawa N. Clinical approaches towards asthma and chronic obstructive pulmonary disease based on the heterogeneity of disease pathogenesis. Clin Exp Allergy 2016; 46: 678-687.

2 Boulet LP, FitzGerald JM, Reddel HK. The revised 2014 GINA strategy report: opportunities for change. Curr Opin Pulm Med 2015; 21: 1-7.

3 Global Strategy for the Diagnosis Management and Prevention of COPD. Global initiative for Chronic Obstructive Lung Disease (GOLD), 2016. Available from: www.goldcopd.org

4 Qaseem A, Wilt TJ, Weinberger SE, et al. Diagnosis and management of stable chronic obstructive pulmonary disease: a clinical practice guideline update from the American College of Physicians, American College of Chest Physicians, American Thoracic Society, and European Respiratory Society. Ann Intern Med 2011; 155: 179-191.

5 Standards for the diagnosis and care of patients with chronic obstructive pulmonary disease. American Thoracic Society. Am J Respir Crit Care Med 1995; 152: S77-S121.

6 de Laurentiis G, Paris D, Melck D, et al. Metabonomic analysis of exhaled breath condensate in adults by nuclear magnetic resonance spectroscopy. Eur Respir J 2008; 32: 1175-1183.

7 Motta A, Paris D, Melck D, et al. Nuclear magnetic resonance-based metabolomics of exhaled breath condensate: methodological aspects. Eur Respir J 2012; 39: 498-500.

8 Bertini I, Luchinat C, Miniati M, et al. Phenotyping COPD by ${ }^{1} \mathrm{H}$ NMR metabolomics of exhaled breath condensate. Metabolomics 2014; 10: 302-311.

9 Motta A, Paris D, D'Amato M, et al. NMR metabolomic analysis of exhaled breath condensate of asthmatic patients at two different temperatures. J Proteome Res 2014; 13: 6107-6120.

10 Maniscalco M, Paris D, Melck DJ, et al. Coexistence of obesity and asthma determines a distinct respiratory metabolic phenotype. J Allergy Clin Immunol 2017; 139: 1536-1547.

11 Eriksson L, Johansson E, Kettaneh-Wold N, et al. Multi and megavariate data analysis. Part I: basic principles and applications. 2nd edn. Umeå, Umetrics AB, 2006.

12 Durham AL, Adcock IM. The relationship between COPD and lung cancer. Lung Cancer 2015; 90: 121-127.

13 Okubo T, Suzuki T, Hosaka M, et al. Effects induced by organic acids in a human lung alveolar carcinoma cell line A549. Yakugaku Zasshi 2016; 136: 1433-1438.

14 Ahmed N, Bezabeh T, Ijare OB, et al. Metabolic signatures of lung cancer in sputum and exhaled breath condensate detected by ${ }^{1} \mathrm{H}$ magnetic resonance spectroscopy: A feasibility study. Magn Reson Insights 2016; 9: 29-35.

15 López-Sánchez LM, Jurado-Gámez B, Feu-Collado N, et al. Exhaled breath condensate biomarkers for the early diagnosis of lung cancer using proteomics. Am J Physiol Lung Cell Mol Physiol 2017; 313: L664-L676. 
16 Traphagen N, Tian Z, Allen-Gipson D. Chronic ethanol exposure: Pathogenesis of pulmonary disease and dysfunction. Biomolecules 2015; 5: 2840-2853.

17 Otsuka M, Mine T, Ohuchi K, et al. A detoxication route for acetaldehyde: metabolism of diacetyl, acetoin, and 2,3-butanediol in liver homogenate and perfused liver of rats. J Biochem 1996; 119: 246-251.

18 Filipiak W, Sponring A, Baur MM, et al. Characterization of volatile metabolites taken up by or released from Streptococcus pneumoniae and Haemophilus influenzae by using GC-MS. Microbiology 2012; 158: 3044-3053.

19 Fens N, Roldaan AC, van der Schee MP, et al. External validation of exhaled breath profiling using an electronic nose in the discrimination of asthma with fixed airways obstruction and chronic obstructive pulmonary disease. Clin Exp Allergy 2011; 41: 1371-1378.

20 Adamko DJ, Sykes BD, Rowe BH. The metabolomics of asthma: novel diagnostic potential. Chest 2012; 141: $1295-1302$.

21 Ghebre MA, Desai D, Singapuri A, et al. Sputum inflammatory mediators are increased in Aspergillus fumigatus culture-positive asthmatics. Allergy Asthma Immunol Res 2017; 9: 177-181.

22 Maniscalco M, Motta A. Biomarkers in allergic asthma: Which matrix should we use? Clin Exp Allergy 2017; 47: 1097-1098. 